\title{
Electronic Behaviors of Individual Defects and Boundaries in 2D Materials: A Spatially Resolved Study with Multi-Probe Scanning Tunneling Microscopy
}

\author{
An-Ping Li
}

\author{
Center for Nanophase Materials Sciences, Oak Ridge National Laboratory, Oak Ridge, TN 37831, USA
}

Electronic properties at the nanoscale are the key to the novel applications of low-dimensional materials in electronic and energy technologies. Due to the restricted dimensionality, one distinctive character of these nano-systems is that the electronic properties are critically dependent on the structural and compositional heterogeneities such as defects and boundaries. Therefore, an important aspect of nanoresearch is to examine the role of defects and boundaries, particularly the correlation between defect structures and electronic properties.

In this regard, multi-probe scanning tunneling microscopy (STM) is a unique enabling tool, as it combines 4 STMs with a scanning electron microscope (SEM) in a well-controlled sample environment to allow for simultaneous measurements of STM and transport across individual heterogeneities. In the last decade, several groups have developed four-probe STM that can perform four-point electrical measurements with probe spacing down to the microscopic scale [1, 2]. By utilizing this unique tool, extensive research has been undertaken to explore aspects of nanotransport, which include (a) intertwined electronic and structural phase transitions in surface supported two-dimensional structures $[1,4]$, (b) effects of atomic defects and interwire coupling on the electronic and transport properties of ultra thin quantum wire systems [5], (c) grain boundary resistances in copper nanowires with one-to-one correspondence to the grain boundary structure [6], and (d) defect scattering effects in two-dimensional electron gas systems [7-10].

2D materials are a silent example, where the presence of defects and boundaries can largely determine the physical properties by dictating the electron scattering, transport, and excitation processes. Here we introduce our recent results on the study of two types of defect in 2D materials to illustrate how electronic and transport properties must be understood with a correlation to the atomic structures. The first type of defect is 1D boundary in hexagonal boron nitride $(\mathrm{hBN})$ and graphene planar heterostructures, where a polar-on-nonpolar 1D interface is expected to possess peculiar electronic states associated with edge states of graphene and the polarity of hBN. By implementing the concept of epitaxy to 2D space, we grew monolayer hBN from fresh edges of monolayer graphene with lattice coherence, forming an atomically sharp 1D boundary [1]. STM/STS measurements reveal a zigzag oriented boundary, showing boundary states about $0.6 \mathrm{eV}$ below or above the Fermi level depending on the terminations of the hBN at the boundary [2]. The boundary states are extended along the boundary, and exponentially decay into the bulk of graphene and hBN. The origin of boundary states and the effect of the polarity discontinuity at the interface will be discussed. Another type of defect is the monolayerbilayer (ML-BL) boundaries in epitaxial graphene on SiC. By measuring the transport spectroscopy across individual ML-BL graphene boundaries with multi-probe scanning tunneling potentiometry, a greater voltage drop is observed when the current flows from bilayer to monolayer graphene than in the reverse direction, displaying an asymmetric electron transport upon bias polarity reversal [3, 4]. Interestingly, this asymmetry is not from a typical nonlinear conductance due to electron transmission through an asymmetric potential. Rather, it indicates the opening of an energy gap at the Fermi energy, a correlation effect that could occur in other systems such as topological insulators as well. [11] 
References:

[1] S Hasegawa et al., Surf. Rev. Lett. 10 (2003), p. 963.

[2] TH Kim, et al, Rev. Sci. Instrum. 78 (2007), p. 123701.

[3] A-P Li et al, Adv. Funct. Mater. 23 (2013), p. 2509.

[4] TH Kim, et al, Proc. Natl. Acad. Sci. USA 107 (2010), p. 5272.

[5] S Qin et al, Nano Lett. 12 (2012), p.938.

[6] TH Kim et al, Nano Lett. 10 (2010), p. 3096.

[7] KW Clark, et al, ACS Nano 7 (2013), p. 7956.

[8] L Liu, et al, Science 343, 163 (2014).

[9] J Park et al, Nature Commun. 5 (2014), p. 5403.

[10] KW Clark, et al, Phys. Rev. X 4 (2014), p. 011021.

[11] This research was conducted at the Center for Nanophase Materials Sciences, which is a DOE Office of Science User Facility.

(a)

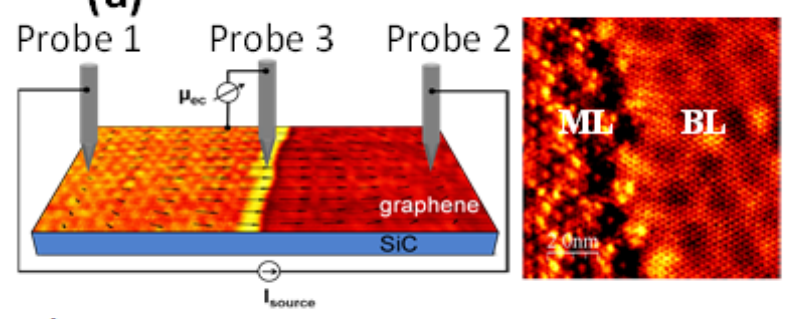

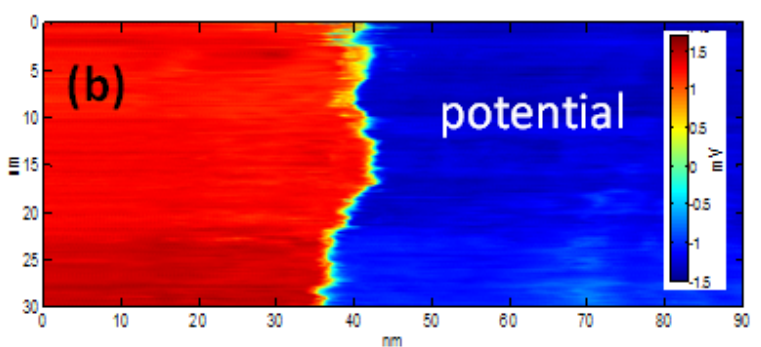

Figure 1. The atomic structure and electrochemical potential distributions measured with a multi-probe STM across a monolayer-bilayer graphene boundary.
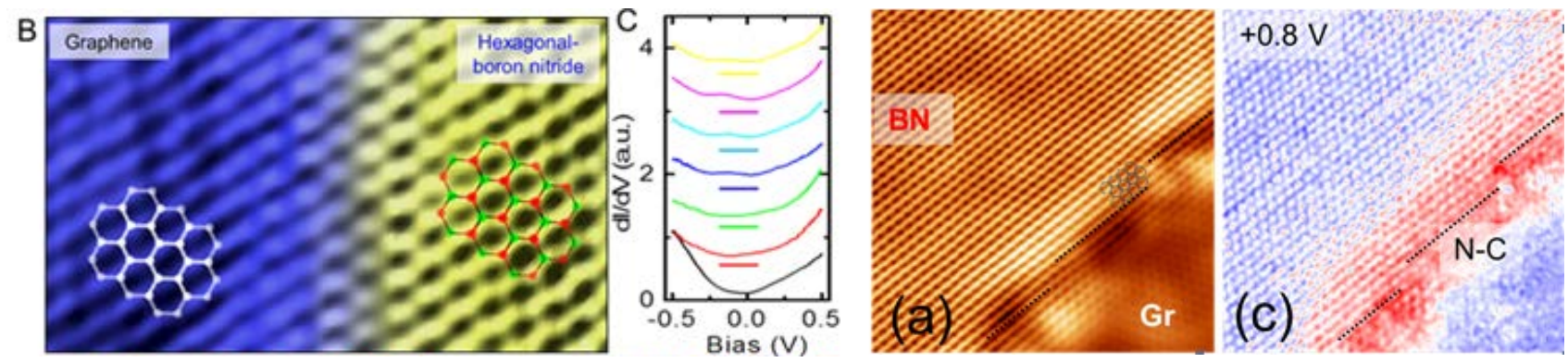

Figure 2. The atomic structure and boundary states at the interface of in-plane graphene-hexagonal BN monolayer junction.. 